\title{
Modelling the Impact of Undetected Cases on the Transmission Dynamics of COVID-19
}

\author{
Herieth Rwezaura \\ Department of Mathematics, University of Dar es Salaam, P.O. Box 35062, Dar es Salaam, \\ Tanzania \\ E-mail: rwezaula.herieth@udsm.ac.tz
}

Received 6 Oct 2021, Revised 24 Dec 2021, Accepted 27 Dec 2021, Published Dec 2021

DOI: https://dx.doi.org/10.4314/tjs.v47i5.25

\begin{abstract}
The spread of COVID-19 globally has caused negative impacts to the public, making understanding the dynamics of transmission a necessity. Essential factors such as undetected cases, asymptomatic cases, and several non-pharmaceutical interventions have played significant roles in the spreading mechanism of COVID-19 in the human population. It is imperative to understand the significance of these factors in order to determine whether COVID-19 will be eradicated or will continue to persist in the population. A mathematical model is formulated to investigate the impacts of vaccination and several non-pharmaceutical interventions on the dynamics of a COVID-19 accounting for asymptomatic cases, detected (identified) and undetected (unidentified) symptomatic infected cases. Results show that vaccination at higher rate, infection detection and immediate quarantine or isolation of infected individuals have the potential to eradicate COVID-19 from the population. It is recommended that individuals should be encouraged to get vaccinated while the government should encourage (or enforce through persuasive communication) the use of non-pharmaceutical interventions such as face masks wearing.
\end{abstract}

Keywords: COVID-19, undetected, vaccination, vaccine efficacy, stability.

\section{Introduction}

Corona virus disease 2019 (COVID-19) is an infectious disease that emerged in Wuhan City of Hubei Province, China in December 2019 and that has rapidly raged in China and subsequently all over the world (Huo et al. 2021). On 30 January 2020, the World Health Organization (WHO) declared the disease to be a Public Health Emergency of international concerns, and then as a pandemic on 11 March 2020. By 19 September 2021, the cumulative numbers of confirmed cases and deaths globally were nearly 228 million and over 4.6 million, respectively, according to the WHO. In Tanzania for instance, with its constrained healthcare resources, from 3 January 2020 to 28 September 2021 there have been 25,674 confirmed cases with 714 deaths reported to $\mathrm{WHO}$ and as of 23 September 2021, a total of 389,807 vaccine doses have been administered.

Essential factors such as undetected cases, asymptomatic cases, and several nonpharmaceutical interventions have played significant roles in the spreading mechanism of COVID-19. Given the rapid spread of COVID-19 globally, it is imperative to understand the significance of these factors to determine whether COVID-19 will be eradicated or will continue to persist in the population.

Results from a recent study by Melis and Littera (2021) noted that the spread of the COVID-19 pandemic is mostly caused by undetected carriers highlighting the significant roles that they play in the 
transmission dynamics of COVID-19. In fact, the speed at which an epidemic grows cannot be explained if we only consider the number of recorded infected patients who, supposedly, are immediately removed from the circulating population by hospitalization or self-isolation.

The world continues to witness that despite massive efforts to mitigate the spread of COVID-19 including introduction of vaccine leave alone various control measures, governments and health decision makers and implementers are continuing to face various challenges including optimal policies for vaccination. Mathematical simulations have long been used to gain insights into the mechanisms of disease transmission, and that the essence of modeling lies in defining a set of equations that mimic the complex transmission dynamics of diseases (Beigi et al. 2021). Since the onset of the epidemic, various mathematical models of COVID-19 abound in the literature (Aldila et al. 2021, Diagne et al. 2021, Mwalili et al. 2020, Tchoumi et al. 2021). In this paper, a mathematical model is formulated to predict the spread of COVID-19 considering asymptomatic cases, detected (identified) and undetected (unidentified) symptomatic infected cases, impact of vaccination and several non-pharmaceutical interventions to mitigate the spread of COVID-19.

\section{Materials and Methods Model formulation}

A deterministic compartmental modelling approach is used to describe the disease transmission dynamics and a homogeneously mixing population is considered where individuals in the population have equal probability of contact with each other. Vaccination strategy to minimize the probability of disease transmission is accounted for as well as detected (identified) and undetected (unidentified) symptomatic cases. To accommodate these factors, at any time $t$, the total population $N(t)$ is subdivided into seven compartments depending on individuals' disease status as follows: susceptible $S(t)$, vaccinated $V(t)$, exposed $E(t)$, asymptomatic having no clinical symptoms but can infect susceptible people $\quad A(t), \quad$ detected (identified) symptomatic infected $I_{D}(t)$, undetected (unidentified) symptomatic infected $I_{U}(t)$, and recovered $R(t)$. The total population $N(t)$ is given by

$N(t)=S(t)+V(t)+E(t)+A(t)+I_{D}(t)+I_{U}(t)+R(t)$.

Susceptible population $S(t)$, is increased by recruiting individuals into the population at a rate $\Lambda$, and through a proportion of vaccinated and recovered individuals that return to susceptible compartment after losing their immunity to the virus at the rate $\omega$ and $\eta$, respectively. This population is decreased because of either vaccination of individuals at a rate $v$, or through infections induced by the disease with the force of infection $\lambda$. Infection with COVID-19 is acquired via effective contacts with infected (contagious) individuals or direct contact with infectious individual contaminants or droplets. The force of infection is given as

$$
\lambda=\frac{\beta\left(\xi_{1} E+\xi_{2} A+\xi_{3} I_{D}+I_{U}\right)}{N},
$$

where $\xi_{1}, \xi_{2}$ and $\xi_{3}$ are modification parameters to reduce infectiousness of exposed, asymptomatic, and detected (identified) symptomatic individuals. The parameter $\xi_{3}$ is associated with precautions like mask wearing, physical distancing, handwashing, and the hygiene consciousness.

Vaccination is given only to susceptible individuals at the rate $v$, which will transfer them into $V(t)$. It is assumed that the vaccine has a validity period of $\omega^{-1}$ and does not protect people perfectly from COVID-19 infections (because the COVID-19 vaccine does not provide $100 \%$ prevention against infections). Thus, vaccinated individual may get infected by COVID-19, but the transmission rate $\beta$ is reduced by $(1-\varepsilon)$ 
where $\varepsilon \in(0,1]$ is the efficacy of the vaccine, ( $\varepsilon=1$ represents a vaccine that offers $100 \%$ protection against infection, while $\varepsilon=0$ means the vaccine offers no protection at all). The vaccinated population $V(t)$ is increased by those vaccinated from the susceptible class at the rate $v$. It is decreased by infections following contact with infectious individual at a rate $(1-\varepsilon) \beta$ and by becoming susceptible again after losing their immunity to the virus at the rate $\omega$.

A proportion $\alpha_{A}$, of the exposed individuals who do not develop symptoms after the incubation period exits by progressing to asymptomatic population $A(t)$ at the rate $\vartheta$. A proportion $\alpha_{D}$, of the exposed individuals who develop symptoms and are detected move to $I_{D}(t)$ class at the rate $\vartheta$. It is assumed that the detected symptomatic infected individuals are isolated and move to the hospital or Intensive Care Units (ICU) immediately until they get recovered from COVID-19. Hence, we assume that the detected symptomatic individuals do not spread disease or have very minimum probability of disease transmission (disease transmission rate/ infections reduced by $\left.\xi_{3}\right)$. The remaining proportion $\alpha_{U}$, of the exposed individuals who develop symptoms and are undetected (unidentified) after the incubation period move to undetected (unidentified) symptomatic class $I_{U}(t)$ at the rate $\vartheta$. Thus, $\left(\alpha_{A}, \alpha_{D}, \alpha_{U}\right) \in(0,1)$ and $\alpha_{A}+\alpha_{D}+\alpha_{U}=1$.
Asymptomatic population $A(t)$ is increased by a proportion $\alpha_{A}$, of the exposed individuals who do not develop symptoms after the incubation period, and they exit the compartment through natural recovery at the rate $\tau_{A}$. The detected symptomatic compartment $I_{D}(t)$ gains population from a proportion $\alpha_{D}$, of the exposed individuals who develop symptoms and are detected after the incubation period, and they exit the compartment through recovery at the rate $\tau_{D}$, or disease-induced death rate $\delta_{1}$. Furthermore, the undetected infected compartment $I_{U}(t)$ increases population from a proportion $\alpha_{U}$, of the exposed individuals who develop symptoms and are undetected after the incubation period. It decreases when individuals exit the compartment through recovery at the rate $\tau_{U}$, or disease-induced death rate $\delta_{2}$.

The recovered compartment $R(t)$, gains population from the asymptomatic, detected infected and undetected infected individuals at the rates $\tau_{A}, \tau_{D}$ and $\tau_{U}$, respectively. The recovered individuals are assumed to develop immunity to COVID-19 for a mean duration $\eta^{-1}$, before they become susceptible again. Furthermore, all individuals in each compartment are assumed to exit their compartments through natural death at the rate $\mu$. A flow diagram of the dynamics of the proposed model is shown in Figure 1, and the model parameter values together with their description and source are presented in Table 1. 


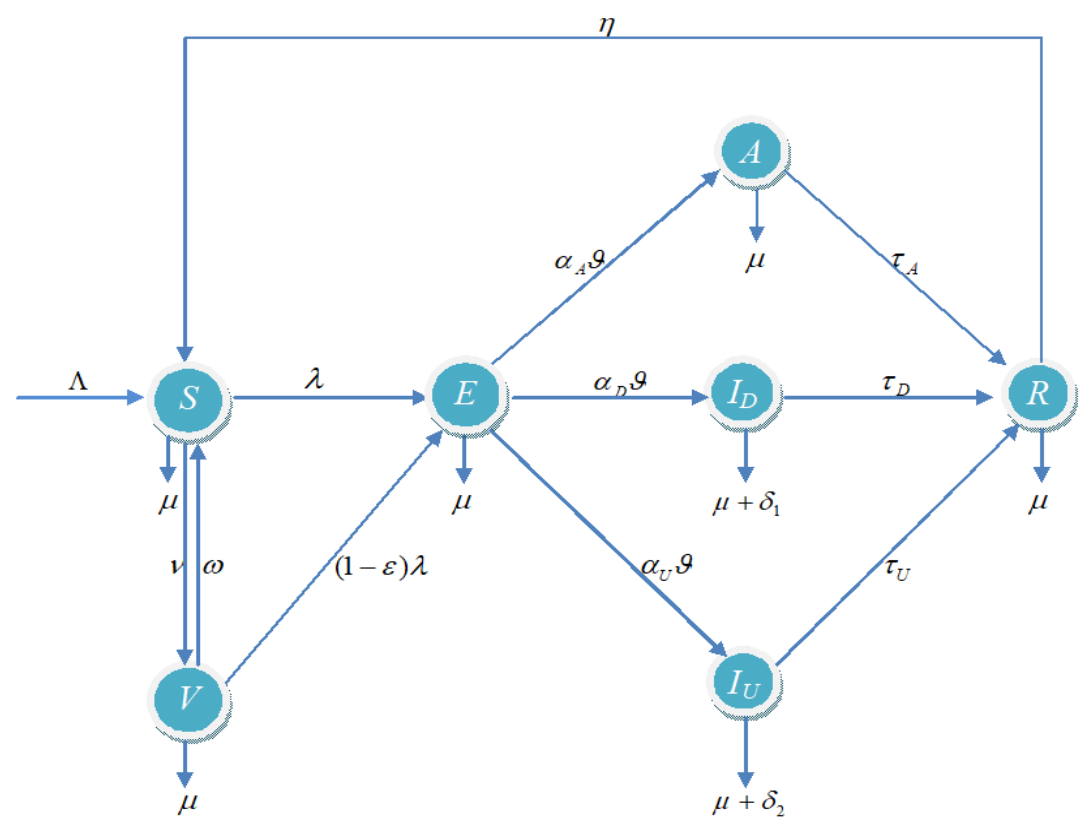

Figure 1: The flow diagram of the COVID-19 model.

From the flow diagram in Figure 1, the model equations are derived as follows:

$$
\begin{aligned}
& \frac{d S}{d t}=\Lambda-(\lambda+v+\mu) S+\omega V+\eta R, \\
& \frac{d V}{d t}=v S-((1-\varepsilon) \lambda+\mu+\omega) V, \\
& \frac{d E}{d t}=\lambda(S+(1-\varepsilon) V)-(\vartheta+\mu) E, \\
& \frac{d A}{d t}=\alpha_{A} \vartheta E-\left(\tau_{A}+\mu\right) A, \\
& \frac{d I_{D}}{d t}=\alpha_{D} \vartheta E-\left(\tau_{D}+\mu+\delta_{1}\right) I_{D}, \\
& \frac{d I_{U}}{d t}=\alpha_{U} \vartheta E-\left(\tau_{U}+\mu+\delta_{2}\right) I_{U}, \\
& \frac{d R}{d t}=\tau_{A} A+\tau_{D} I_{D}+\tau_{U} I_{U}-(\mu+\eta) R,
\end{aligned}
$$

with initial conditions

$S(0) \geq 0, V(0) \geq 0, E(0) \geq 0, A(0) \geq 0, I_{D}(0) \geq 0, I_{U}(0) \geq 0, R(0) \geq 0$,

where $\lambda=\frac{\beta\left(\xi_{1} E+\xi_{2} A+\xi_{3} I_{D}+I_{U}\right)}{N}$, and $N=S+V+E+A+I_{D}+I_{U}+R$. 
Table 1: Description of model parameters

\begin{tabular}{|c|c|c|c|}
\hline Parameter & Description & Value & Source \\
\hline$\Lambda$ & Recruitment & 10000 & Diagne et al. (2021), \\
\hline & individuals into the population & $\overline{59 \times 365}$ & Tchoumi et al. (2021) \\
\hline$\mu$ & Natural death rate & $\hat{1}$ & Aldila et al. (2021) \\
\hline$v$ & Vaccination rate & $\begin{array}{c}65 \times 365 \\
0.02\end{array}$ & Assumed \\
\hline$\beta$ & Transmission rate & 0.4531 & Tchoumi et al. (2021) \\
\hline$\varepsilon$ & Efficacy of the vaccine & 0.8 & Aldila et al. (2021) \\
\hline$\xi_{1}$ & $\begin{array}{l}\text { Modification parameter to } \\
\text { reduce infectiousness } \\
\text { exposed individual }\end{array}$ & 0.3 & Chinwendu et al. (2020) \\
\hline$\xi_{2}$ & $\begin{array}{l}\text { Modification parameter to } \\
\text { reduce infectiousness } \\
\text { asymptomatic individual }\end{array}$ & $0.2-0.3$ & Assumed \\
\hline$\xi_{3}$ & $\begin{array}{l}\text { Modification parameter to } \\
\text { reduce infectiousness } \\
\text { detected symptomatic }\end{array}$ & 0.1 & Chinwendu et al. (2020) \\
\hline$\vartheta$ & $\begin{array}{l}\text { Rate of progression from } \\
\text { exposed state to infectious } \\
\text { state }\end{array}$ & 0.13 & $\begin{array}{l}\text { Tang et al. (2020), } \\
\text { Diagne et al. (2021) }\end{array}$ \\
\hline$\alpha_{A}$ & $\begin{array}{l}\begin{array}{l}\text { Proportion of } \\
\text { individuals who } \\
\text { asymptomatic }\end{array} \\
\begin{array}{l}\text { become } \\
\text { whosed }\end{array}\end{array}$ & 0.3 & $\begin{array}{l}\text { CDC (2021), Mwalili et } \\
\text { al. (2020), Diagne et al. } \\
\text { (2021) }\end{array}$ \\
\hline$\alpha_{D}$ & $\begin{array}{l}\text { Proportion of exposed } \\
\text { individuals who become } \\
\text { detected symptomatic }\end{array}$ & 0.3 & Assumed \\
\hline$\alpha_{U}$ & $\begin{array}{l}\text { Proportion of exposed } \\
\text { individuals who become } \\
\text { undetected symptomatic }\end{array}$ & 0.4 & Assumed \\
\hline$\tau_{A}$ & $\begin{array}{l}\text { The recovery rate for } \\
\text { infectious asymptomatic } \\
\text { individuals }\end{array}$ & 0.0714 & Mwalili et al. (2020) \\
\hline$\tau_{D}$ & $\begin{array}{l}\text { The recovery rate for } \\
\text { infectious detected } \\
\text { symptomatic individuals }\end{array}$ & 0.0701 & Diagne et al. (2021) \\
\hline$\tau_{U}$ & $\begin{array}{l}\text { The recovery rate for } \\
\text { infectious undetected } \\
\text { symptomatic individuals }\end{array}$ & 0.05 & Mwalili et al. (2020) \\
\hline$\delta_{1}$ & $\begin{array}{l}\text { Disease-induced death rate for } \\
\text { detected } \\
\text { individuals }\end{array}$ & 0.018 & Diagne et al. (2021) \\
\hline$\delta_{2}$ & $\begin{array}{l}\text { Disease-induced death rate for } \\
\text { undetected } \\
\text { individuals }\end{array}$ & 0.018 & Diagne et al. (2021) \\
\hline$\eta$ & $\begin{array}{l}\text { Rate at which individuals lose } \\
\text { immunity after recovery }\end{array}$ & 0.011 & $\begin{array}{l}\text { Shakhany and } \\
\text { Salimifard (2021), } \\
\text { Diagne et al. (2021) }\end{array}$ \\
\hline$\omega$ & $\begin{array}{l}\text { Rate at which vaccinated } \\
\text { individuals lose immunity } \\
\text { (Vaccine waning immunity) }\end{array}$ & 0.004 & Assumed \\
\hline
\end{tabular}




\section{Model analysis}

Invariant region: The solutions of the model (1) are uniformly bounded in a positive invariant region, $\Omega=\left\{\left(S(t), V(t), E(t), A(t), I_{D}(t), I_{U}(t), R(t)\right) \in \mathbb{R}_{+}^{7}: N \leq \frac{\Lambda}{\mu}\right\}$.

Proof: Let, $\left(S(t), V(t), E(t), A(t), I_{D}(t), I_{U}(t), R(t)\right) \in \mathbb{R}_{+}^{7}$ be any solution of the system with non-negative initial conditions. Then, adding the differential equations in the model system (1) gives $\frac{d N}{d t}=\Lambda-\mu N-\left(\delta_{1} I_{D}+\delta_{2} I_{U}\right) \leq \Lambda-\mu N$.

The given initial conditions

$S(0) \geq 0, V(0) \geq 0, E(0) \geq 0, A(0) \geq 0, I_{D}(0) \geq 0, I_{U}(0) \geq 0, R(0) \geq 0$, ensure that $N(0) \geq 0$.

Using a standard comparison theorem (Lakshmikantham et al. 1989) we can show that $N(t) \leq N(0) e^{-\mu t}+\frac{\Lambda}{\mu}\left(1-e^{-\mu t}\right)$.

In particular, $N(t) \leq \frac{\Lambda}{\mu}$ if $N(0) \leq \frac{\Lambda}{\mu}$. Thus, the region is positively invariant. Hence, it is sufficient to consider the dynamics of the flow generated by (1) in $\Omega$. In this region, the model is epidemiologically and mathematically well-posed (Hethcote 2000). Thus, every solution of the model (1) with initial conditions in $\Omega$ remains in $\Omega$ for all $t>0$.

\section{Positivity and boundedness of solutions}

Positivity of solutions: Since the system of equations (1) represents human populations, all parameters in the model are non-negative and it can be shown that, given non-negative initial values, the solutions of the system are non-negative. The following lemma proves that the solution of the model is nonnegative for $t \geq 0$.

\section{Lemma 1}

If $S(0) \geq 0, V(0) \geq 0, E(0) \geq 0, A(0) \geq 0, I_{D}(0) \geq 0, I_{U}(0) \geq 0$ and $R(0) \geq 0$, then the solutions $\left(S(t), V(t), E(t), A(t), I_{D}(t), I_{U}(t), R(t)\right)$ of system (1) remain non-negative for all $t>0$.

Proof: From the first equation of system (1), $\frac{d S}{d t}=\Lambda-(\lambda+v+\mu) S+\omega V+\eta R \geq-(\lambda+v+\mu) S$,

which upon integration yields $S(t) \geq S(0) \exp \left(-\int_{0}^{t}(\lambda+v+\mu) d t\right)>0$.

Hence, $S(t)$ remains non-negative for all $t>0$. In the same way, it can be shown that the other equations of system (1) are also positive for all $t>0$. Therefore, this concludes that, the solutions of system (1) are positive (non-negative) for all values of $t>0$.

\section{Boundedness of the model}

Lemma 2 All solutions of the model system (1) with non-negative initial conditions are bounded and $N(t) \leq \frac{\Lambda}{\mu}$ for all $t>0$.

Proof: Adding the differential equations in the model system (1) gives 


$$
\frac{d N}{d t}=\Lambda-\mu N-\left(\delta_{1} I_{D}+\delta_{2} I_{U}\right) \Rightarrow \frac{d N}{d t} \leq \Lambda-\mu N
$$

Lemma 1 ensure that $N(t) \geq 0$. Thus, the total population $N(t)$ is positive for all $t>0$.

Clearly $\lim _{t \rightarrow \infty} \sup N(t) \leq \frac{\Lambda}{\mu}$. Hence, $0 \leq N(t) \leq \frac{\Lambda}{\mu}$ for all $t>0$. This concludes that, all solutions of the model (1) are bounded.

\section{Computation of the reproduction numbers $R_{0}$ and $R_{V}$}

The threshold parameter $R_{0}$ is the basic reproduction number of system (1) where no vaccine intervention is implemented (it represents the number of secondary infections generated by a single infectious case in a totally susceptible population), while $R_{V}$ is the effective reproduction number that can be interpreted as the number of infections generated by one infected individual introduced into a completely naive population when vaccination is being implemented. The model (1) has a disease-free equilibrium (DFE) given by, $E^{0}=\left(\frac{\Lambda(\omega+\mu)}{\mu(v+\omega+\mu)}, \frac{v \Lambda}{\mu(v+\omega+\mu)}, 0,0,0,0,0,0\right)$.

The threshold quantity, $R_{V}$ is computed using the next generation operator of van den Driessche and Watmough (2002), and it is obtained as the spectral radius of the matrix $F V^{-1}$ at the DFE $E^{0}$ with $F$ and $V$, respectively given by

$$
F=\left(\begin{array}{cccc}
\frac{\beta \xi_{1}(\omega+\mu+(1-\varepsilon) v)}{\omega+\mu+v} & \frac{\beta \xi_{2}(\omega+\mu+(1-\varepsilon) v)}{\omega+\mu+v} & \frac{\beta \xi_{3}(\omega+\mu+(1-\varepsilon) v)}{\omega+\mu+v} & \frac{\beta(\omega+\mu+(1-\varepsilon) v)}{\omega+\mu+v} \\
0 & 0 & 0 & 0 \\
0 & 0 & 0 & 0 \\
0 & 0 & 0 & 0
\end{array}\right)
$$

and

$$
V=\left(\begin{array}{cccc}
\vartheta+\mu & 0 & 0 & 0 \\
-\alpha_{A} \vartheta & \tau_{A}+\mu & 0 & 0 \\
-\alpha_{D} \vartheta & 0 & \tau_{D}+\mu+\delta_{1} & 0 \\
-\alpha_{U} \vartheta & 0 & 0 & \tau_{U}+\mu+\delta_{2}
\end{array}\right)
$$

It follows that the effective reproduction number of the model system (1), is given by

$$
R_{V}=\frac{\beta(\omega+\mu+(1-\varepsilon) v)}{(\omega+\mu+v)}\left[\frac{\xi_{1}}{(\vartheta+\mu)}+\frac{\alpha_{A} \vartheta \xi_{2}}{(\vartheta+\mu)\left(\tau_{A}+\mu\right)}+\frac{\alpha_{D} \vartheta \xi_{3}}{(\vartheta+\mu)\left(\tau_{D}+\mu+\delta_{1}\right)}+\frac{\alpha_{U} \vartheta}{(\vartheta+\mu)\left(\tau_{U}+\mu+\delta_{2}\right)}\right] \text {. }
$$

In the absence of vaccination $(\nu=0=\omega)$, we have the basic reproduction number given by

$$
R_{0}=\frac{\beta \xi_{1}}{\vartheta+\mu}+\frac{\beta \alpha_{A} \vartheta \xi_{2}}{(\vartheta+\mu)\left(\tau_{A}+\mu\right)}+\frac{\beta \alpha_{D} \vartheta \xi_{3}}{(\vartheta+\mu)\left(\tau_{D}+\mu+\delta_{1}\right)}+\frac{\beta \alpha_{U} \vartheta}{(\vartheta+\mu)\left(\tau_{U}+\mu+\delta_{2}\right)}
$$


$=R_{E}+R_{A}+R_{I_{D}}+R_{I_{U}}$

The basic reproduction number obtained in (3) clearly breaks down to four components: secondary infections generated from the exposed, infectious asymptomatic, infectious detected and infectious undetected symptomatic individuals, respectively. Note that,

$$
\begin{aligned}
& R_{V}=\frac{(\omega+\mu+(1-\varepsilon) v)}{(\omega+\mu+v)}\left[\frac{\beta \xi_{1}}{\vartheta+\mu}+\frac{\beta \alpha_{A} \vartheta \xi_{2}}{(\vartheta+\mu)\left(\tau_{A}+\mu\right)}+\frac{\beta \alpha_{D} \vartheta \xi_{3}}{(\vartheta+\mu)\left(\tau_{D}+\mu+\delta_{1}\right)}+\frac{\beta \alpha_{U} \vartheta}{(\vartheta+\mu)\left(\tau_{U}+\mu+\delta_{2}\right)}\right] \\
& R_{V}=\frac{(\omega+\mu+(1-\varepsilon) v)}{(\omega+\mu+v)} R_{0}=K R_{0}
\end{aligned}
$$

From the expression of $R_{V}$, it can be observed that $R_{V}<R_{0}$ because $K=\frac{(\omega+\mu+(1-\varepsilon) v)}{(\omega+\mu+v)}<1$.

The higher the efficacy of the vaccine (large value of $\varepsilon \in(0,1]$ ), the smaller is the value of $K$. The parameter $K$ represents the effect of vaccine implementation in reducing the initial basic reproduction number, which depends on the rate of vaccination and quality (efficacy) of the vaccine. Hence, it can be concluded that the implementation of a vaccination at a constant rate $v$ reduces the basic reproduction number by $K$ percent (Aldila et al. 2021).

\section{Stability of the Disease-Free Equilibrium (DFE)}

\section{Local stability of the DFE of COVID-19 model}

Theorem 1 The disease-free equilibrium point $E^{0}$ of model system (1) is locally asymptotically stable if $R_{V}<1$, and unstable if $R_{V}>1$.

Proof: The local stability of the DFE of the COVID-19 model (1) is determined by its effective reproduction number $R_{V}$. The Jacobian matrix of the COVID-19 model system (1) at the DFE $E^{0}$ is given by

$$
J_{E^{0}}=\left(\begin{array}{ccccccc}
-a_{1} & \omega & -\frac{\beta \xi_{1}(\omega+\mu)}{v+\omega+\mu} & -\frac{\beta \xi_{21}(\omega+\mu)}{v+\omega+\mu} & -\frac{\beta \xi_{3}(\omega+\mu)}{v+\omega+\mu} & -\frac{\beta(\omega+\mu)}{v+\omega+\mu} & \eta \\
v & -a_{2} & -\frac{\beta \xi_{1}(1-\varepsilon) v}{v+\omega+\mu} & -\frac{\beta \xi_{2}(1-\varepsilon) v}{v+\omega+\mu} & -\frac{\beta \xi_{3}(1-\varepsilon) v}{v+\omega+\mu} & -\frac{\beta(1-\varepsilon) v}{v+\omega+\mu} & 0 \\
0 & 0 & \frac{\beta \xi_{1}(\omega+\mu+(1-\varepsilon) v)}{\omega+\mu+v} & \frac{\beta \xi_{2}(\omega+\mu+(1-\varepsilon) v)}{\omega+\mu+v} & \frac{\beta \xi_{3}(\omega+\mu+(1-\varepsilon) v)}{\omega+\mu+v} & \frac{\beta(\omega+\mu+(1-\varepsilon) v)}{\omega+\mu+v} & 0 \\
0 & 0 & \alpha_{A} \vartheta & -a_{4} & 0 & 0 & 0 \\
0 & 0 & \alpha_{D} \vartheta & 0 & -a_{5} & 0 & 0 \\
0 & 0 & \alpha_{U} \vartheta & 0 & 0 & -a_{6} & 0 \\
0 & 0 & 0 & \tau_{A} & \tau_{D} & \tau_{U} & -a_{7}
\end{array}\right)
$$

where $a_{1}=v+\mu, a_{2}=\mu+\omega, a_{3}=\vartheta+\mu, a_{4}=\tau_{A}+\mu, a_{5}=\tau_{D}+\mu+\delta_{1}$,

$a_{6}=\tau_{U}+\mu+\delta_{2}$ and $a_{7}=\mu+\eta$. The five eigenvalues are $\lambda_{1}=-(v+\omega+\mu)$, $\lambda_{2}=-\mu, \lambda_{3}=-\left(\tau_{D}+\mu+\delta_{1}\right), \lambda_{4}=-\left(\tau_{U}+\mu+\delta_{2}\right)$, and $\lambda_{5}=-(\mu+\eta)$, while the remaining two eigenvalues are obtained from the $2 \times 2$ matrix

$$
M=\left(\begin{array}{cc}
\frac{\beta \xi_{1}(\omega+\mu+(1-\varepsilon) v)}{\omega+\mu+v}-(\vartheta+\mu) & \frac{\beta \xi_{2}(\omega+\mu+(1-\varepsilon) v)}{\alpha_{A} \vartheta} \\
\omega+\mu+v & -\left(\tau_{A}+\mu\right)
\end{array}\right)
$$


Then, the eigenvalues of $M$ are real and negative if the Routh-Hurwitz condition is satisfied. Applying the Routh-Hurwitz conditions $\operatorname{Tr} M<0$, and Det $M>0$, we have

$$
\begin{aligned}
& \operatorname{Tr} M=\frac{\beta \xi_{1}(\omega+\mu+(1-\varepsilon) v)}{\omega+\mu+v}-(\vartheta+\mu)-\left(\tau_{A}+\mu\right) \\
& =-\frac{1}{\omega+\mu+v}\left[\left(\vartheta+2 \mu+\tau_{A}\right)(\omega+\mu+v)-\beta \xi_{1}(\omega+\mu+(1-\varepsilon) v)\right]<0,
\end{aligned}
$$

$\operatorname{Det} M=-\left(\tau_{A}+\mu\right)\left[\frac{\beta \xi_{1}(\omega+\mu+(1-\varepsilon) v)}{\omega+\mu+v}-(\vartheta+\mu)\right]-\frac{\beta \xi_{2} \alpha_{A} \vartheta(\omega+\mu+(1-\varepsilon) v)}{\omega+\mu+v}$

$$
\begin{aligned}
& =\left[\left(\tau_{A}+\mu\right)(\vartheta+\mu)-\frac{\beta \xi_{1}(\omega+\mu+(1-\varepsilon) v)}{\omega+\mu+v}\left(\tau_{A}+\mu\right)\right]-\frac{\beta \xi_{2} \alpha_{A} \vartheta(\omega+\mu+(1-\varepsilon) v)}{\omega+\mu+v}, \\
& =(\vartheta+\mu)\left(\tau_{A}+\mu\right)\left[1-\frac{\beta \xi_{1}(\omega+\mu+(1-\varepsilon) v)}{(\omega+\mu+v)(\vartheta+\mu)}-\frac{\beta \xi_{2} \alpha_{A} \vartheta(\omega+\mu+(1-\varepsilon) v)}{(\omega+\mu+v)\left(\tau_{A}+\mu\right)(\vartheta+\mu)}\right], \\
& =(\vartheta+\mu)\left(\tau_{A}+\mu\right)\left[1-\frac{\beta(\omega+\mu+(1-\varepsilon) v)}{(\omega+\mu+v)}\left(\frac{\xi_{1}}{(\vartheta+\mu)}+\frac{\xi_{2} \alpha_{A} \vartheta}{\left(\tau_{A}+\mu\right)(\vartheta+\mu)}\right)\right], \\
& >a_{3} a_{4}\left[1-\frac{\beta(\omega+\mu+(1-\varepsilon) v)}{(\omega+\mu+v)}\left(\frac{\xi_{1}}{(\vartheta+\mu)}+\frac{\xi_{2} \alpha_{A} \vartheta}{\left(\tau_{A}+\mu\right)(\vartheta+\mu)}+\frac{\alpha_{D} \vartheta \xi_{3}}{(\vartheta+\mu)\left(\tau_{D}+\mu+\delta_{1}\right)}+\frac{\alpha_{U} \vartheta}{(\vartheta+\mu)\left(\tau_{U}+\mu+\delta_{2}\right)}\right)\right] \\
& =(\vartheta+\mu)\left(\tau_{A}+\mu\right)\left[1-R_{V}\right]>0 \text { if } R_{V}<1 .
\end{aligned}
$$

Hence, following Theorem 2 of van den Driessche and Watmough (2002), it can be concluded that the DFE $E^{0}$ is locally asymptotically stable when $R_{V}<1$, and unstable otherwise.

From Theorem 1, there is a possibility that COVID-19 could be eradicated from the community if $R_{V}<1$. The derivative of the effective reproduction number $R_{V}$ with respect to the transmission rate $\beta$

$$
\frac{\partial R_{V}}{\partial \beta}=\frac{(\omega+\mu+(1-\varepsilon) v)}{(\omega+\mu+v)}\left[\frac{\xi_{1}}{\vartheta+\mu}+\frac{\alpha_{A} \vartheta \xi_{2}}{(\vartheta+\mu)\left(\tau_{A}+\mu\right)}+\frac{\alpha_{D} \vartheta \xi_{3}}{(\vartheta+\mu)\left(\tau_{D}+\mu+\delta_{1}\right)}+\frac{\alpha_{U} \vartheta}{(\vartheta+\mu)\left(\tau_{U}+\mu+\delta_{2}\right)}\right]
$$

is always positive. This implies that reducing the transmission rate could reduce the reproduction number linearly. This could be accomplished through social distancing or lockdowns.

Similarly, the impacts of vaccination to the effective reproduction number $R_{V}$ can be analyzed. Taking the partial derivative of $R_{V}$ with respect to vaccination rate $v$ gives

$$
\frac{\partial R_{V}}{\partial v}=\frac{(1-\varepsilon)(\omega+\mu+v) R_{0}-(\omega+\mu+(1-\varepsilon) v) R_{0}}{(\omega+\mu+v)^{2}}=\frac{-\varepsilon(\omega+\mu) R_{0}}{(\omega+\mu+v)^{2}}
$$

which is always negative. The vaccination rate is inversely proportional to $R_{V}$, this implies that increasing the vaccination rate could reduce the reproduction number. 


\section{Global stability of the DFE of COVID-19- model}

Using the approach of Castillo-Chavez et al. (2002), the model system (1) is rewritten in the form

$\left\{\begin{array}{l}\frac{d \mathbf{x}}{d t}=F(\mathbf{x}, \boldsymbol{I}), \\ \frac{d \boldsymbol{I}}{d t}=G(\mathbf{x}, \boldsymbol{I}), \quad G(\mathbf{x}, \mathbf{0})=0,\end{array}\right.$

where $\mathbf{x} \in \mathbb{R}^{\boldsymbol{m}}$ denotes the number of uninfected individuals and $\boldsymbol{I} \in \mathbb{R}^{\boldsymbol{n}}$ denotes the number of infected individuals including latent, infectious. Moreover, $\boldsymbol{E}^{0}=\left(\mathbf{x}^{*}, 0\right)$ denote the disease-free equilibrium of this system. The conditions (H1) and (H2) below must be met to guarantee global asymptotic stability.

(H1) For $\frac{d \mathbf{x}}{d t}=F(\mathbf{x}, 0), \mathbf{x}^{*}$ is globally asymptotically stable (g.a.s.),

$(\mathrm{H} 2) G(\mathbf{x}, I)=A I-\hat{G}(\mathbf{x}, I), \hat{G}(\mathbf{x}, I) \geq 0$ for $(\mathbf{x}, I) \in \Omega$,

where $A=D_{I}\left(\mathbf{x}^{*}, 0\right)$ is an M-matrix (the off diagonal elements of $A$ are nonnegative) and $\Omega$ is the region where the model makes biological sense.

If System (5) satisfies the above two conditions, then the following theorem holds:

Theorem 2: The fixed point $\boldsymbol{E}^{0}=\left(\mathbf{x}^{*}, 0\right)$ is a globally asymptotic stable (g.a.s.) equilibrium of (5) provided that $R_{0}<1$ (1.a.s.) and that assumptions (H1) and (H2) are satisfied.

Proof: (see Castillo-Chavez et al. 2002).

Theorem 3: The DFE $E^{0}$ of model system (1) is globally asymptotically stable if $R_{V}<1$.

Proof: The model system (1) is re-written in the form of (5) by setting $\boldsymbol{X}=(S, V)$,

$$
I=\left(E, A, I_{D}, I_{U}, R\right), \boldsymbol{E}^{0}=\left(\mathbf{x}^{*}, \mathbf{0}\right)=\left(\frac{\Lambda(\omega+\mu)}{\mu(v+\omega+\mu)}, \frac{v \Lambda}{\mu(v+\omega+\mu)}, 0\right) \text { and }
$$

the system

$$
\frac{d \mathbf{x}}{d t}=F(\mathbf{x}, 0) \text { becomes }\left\{\begin{array}{l}
\dot{S}=\Lambda-(v+\mu) S+\omega V \\
\dot{V}=v S-(\mu+\omega) V
\end{array},\right.
$$

This equation has a unique equilibrium point

$$
\mathbf{x}^{*}=\left(\frac{\Lambda(\omega+\mu)}{\mu(v+\omega+\mu)}, \frac{v \Lambda}{\mu(v+\omega+\mu)}\right)
$$

which is globally asymptotically stable. Therefore, the condition (H1) is satisfied.

The second condition (H2) can now be verified. The model system (1), has

$$
G(\boldsymbol{x}, \boldsymbol{I})=\left(\begin{array}{c}
\lambda(S+(1-\varepsilon) V)-(\vartheta+\mu) E \\
\alpha_{A} \vartheta E-\left(\tau_{A}+\mu\right) A \\
\alpha_{D} \vartheta E-\left(\tau_{D}+\mu+\delta_{1}\right) I_{D} \\
\alpha_{U} \vartheta E-\left(\tau_{U}+\mu+\delta_{2}\right) I_{U} \\
\tau_{A} A+\tau_{D} I_{D}+\tau_{U} I_{U}-(\mu+\eta) R
\end{array}\right),
$$




$$
D_{I}\left(\boldsymbol{x}^{*}, \mathbf{0}\right)=\left(\begin{array}{ccccc}
\frac{\beta \xi_{1}}{N^{0}} S^{0}+\frac{(1-\varepsilon) \beta \xi_{1}}{N^{0}} V^{0}-a_{3} & \frac{\beta \xi_{2}}{N^{0}} S^{0}+\frac{(1-\varepsilon) \beta \xi_{2}}{N^{0}} V^{0} & \frac{\beta \xi_{3}}{N^{0}} S^{0}+\frac{(1-\varepsilon) \beta \xi_{3}}{N^{0}} V^{0} & \frac{\beta}{N^{0}} S^{0}+\frac{(1-\varepsilon)}{N^{0}} V^{0} & 0 \\
\alpha_{A} \vartheta & -a_{4} & 0 & 0 & 0 \\
\alpha_{D} \vartheta & 0 & -a_{5} & 0 & 0 \\
\alpha_{U} \vartheta & 0 & 0 & -a_{6} & 0 \\
0 & \tau_{A} & \tau_{D} & \tau_{U} & -a_{7}
\end{array}\right)
$$

where $a_{3}=\vartheta+\mu, a_{4}=\tau_{A}+\mu, a_{5}=\tau_{D}+\mu+\delta_{1}, a_{6}=\tau_{U}+\mu+\delta_{2}$ and $a_{7}=\mu+\eta$.

Clearly, $A=D_{I}\left(\mathbf{x}^{*}, 0\right)$ is an M-matrix (the off-diagonal elements of $A$ are nonnegative). On the other hand

$$
\hat{G}(\boldsymbol{x}, \boldsymbol{I})=A I-G(\boldsymbol{x}, \boldsymbol{I})=\left(\beta\left(\xi_{1} E+\xi_{2} A+\xi_{3} I_{D}+I_{U}\right)\left(\frac{S^{0}+(1-\varepsilon) V^{0}}{N^{0}}-\frac{S+(1-\varepsilon) V}{N}\right)\right),
$$

Thus $\hat{G}(\boldsymbol{x}, \boldsymbol{I}) \geq 0$ for all $(\boldsymbol{x}, \boldsymbol{I}) \in \Omega$, furthermore the conditions (H1) and (H2) are satisfied. By Theorem 2, the global stability of the DFE is obtained, thereby completing the proof for the global stability of the DFE $E^{0}$ of the model system (1).

Global stability of the DFE precludes the model system (1) to exhibit bi-stability also known as backward bifurcation (CastilloChavez and Song 2004, Dushoff et al. 1998), a situation where both the disease-free and endemic equilibria coexist when $R_{V}<1$. Since the DFE is globally asymptotically stable, the endemic equilibrium which exists when R_V $>1$ will also be globally asymptotically stable.

\section{Sensitivity analysis}

The sensitivity analysis describes how the model parameters influence effective reproduction number $R_{V}$ as well as the disease transmission. Sensitivity indices allow us to measure how important each parameter is to disease transmission, while its analysis is mainly used to determine the robustness of model prediction to the parameter values (since there are usually errors in the data collection and presumed parameter values).
The sensitivity indices to the parameters in the model are calculated in order to determine parameters that have a high impact on $R_{V}$ and that should be targeted by intervention strategies (Chitnis et al. 2008). Therefore, the sensitivity analysis on the effective reproductive number $R_{V}$ to the parameters in the model are computed to quantify the variations in the model parameters and to identify the most critical parameters (that have a high impact on $R_{V}$ as well as on the disease transmission) that will curtail the spread of COVID-19. In computing the sensitivity analysis, the approach described by Chitnis et al. (2008) is used. The normalized forward sensitivity index of $R_{V}$, that depends differentiably on a parameter $p$, is defined as $\Upsilon_{p}^{R_{V}}=\frac{\partial R_{0}}{\partial p} \times \frac{p}{R_{0}}$, where $\Upsilon_{p}^{R_{0}}$ is the sensitivity index of $R_{V}$ with respect to parameter $p$. Table 2 and Figure 2 show the sensitivity indices of the effective reproduction number $R_{V}$ with respect to each of the parameters related to $R_{V}$ for the model system (1). From Table 2, the sensitivity indices with negative signs indicate that the value of $R_{V}$ decreases when the parameter values are increased and the value of $R_{V}$ increases when the parameter values are decreased, while sensitivity indices 
with positive signs indicate that the value of $R_{V}$ increases when the parameter values are increased and the value of $R_{V}$ decreases when the parameter values are decreased. The most positive index is $\beta$ which implies that increasing (decreasing) of $\beta$ by $10 \%$ will also increase (decrease) the value of $R_{V}$ by $10 \%$. Thus, the increment (decrement) of any proportion of the amount in the transmission rate $\beta$ will also increase (decrease) $R_{V}$ by the same proportion. Therefore, as transmission rate gets lower, the disease also vanishes from the community. The most negative sensitive parameter is $\varepsilon$, which implies that increasing (decreasing) of $\varepsilon$ by $10 \%$ will also decrease (increase) the value of $R_{V}$ by $19.8952 \%$. This suggests that increasing the vaccine efficacy will halt the spread of COVID-19 (the higher the vaccine efficacy will give the rapid reduction in the effective reproduction number $R_{V}$ ). Therefore, the effective reproduction number $R_{V}$ can be controlled by reducing the disease transmission rate $\beta$ and by increasing the vaccine efficacy $\varepsilon$ which has been shown by contour plot in the Figure 3 (The impact of the disease transmission rate $\beta$ and vaccine efficacy $\varepsilon$ on $R_{V}$ ).

Table 2: Sensitivity indices of the effective reproduction number $R_{V}$ to parameters for the COVID-19 model, evaluated at the baseline parameter values listed in the Table 1

\begin{tabular}{lccccc}
\hline Parameter & $\mu$ & $v$ & $\beta$ & $\varepsilon$ & $\xi_{1}$ \\
\hline Sensitivity index & 0.00270 & -0.33449 & 1 & -1.98952 & 0.24097 \\
\hline Parameter & $\xi_{2}$ & $\xi_{3}$ & $\vartheta$ & $\alpha_{A}$ & $\alpha_{D}$ \\
\hline Sensitivity index & 0.10962 & 0.03554 & -0.24065 & 0.10962 & 0.03554 \\
\hline Parameter & $\alpha_{U}$ & $\tau_{A}$ & $\tau_{D}$ & $\tau_{U}$ & $\delta_{1}$ \\
\hline Sensitivity index & 0.61386 & -0.10956 & -0.02827 & -0.45109 & -0.00726 \\
\hline Parameter & $\delta_{2}$ & $\omega$ & & & \\
\hline Sensitivity index & -0.16239 & 0.3310 & & & \\
\hline
\end{tabular}

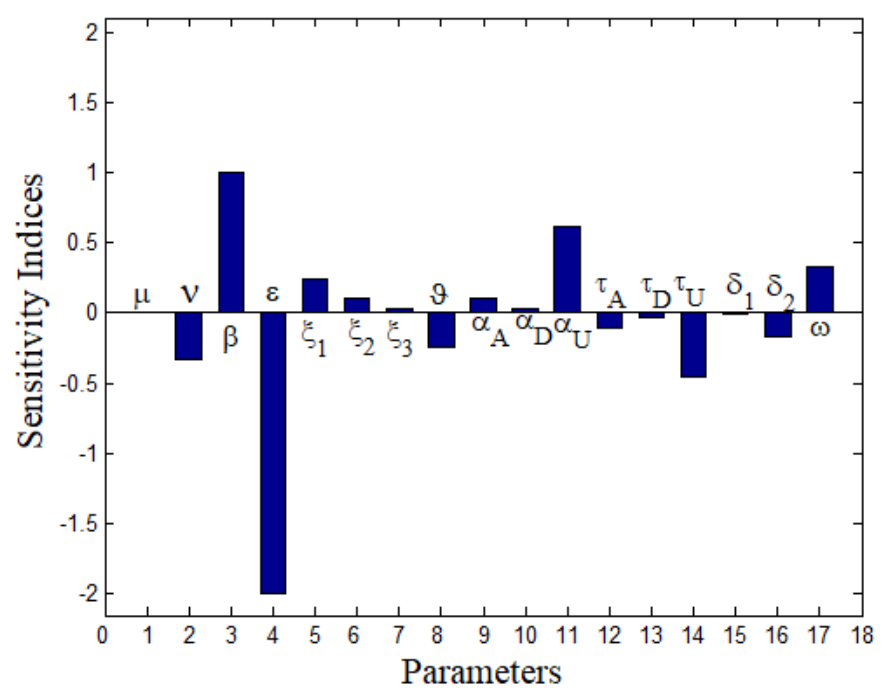

Figure 2: Sensitivity indices of the effective reproduction number $R_{V}$ with respect to each of the system parameters related to $R_{V}$ for the model system (1). 


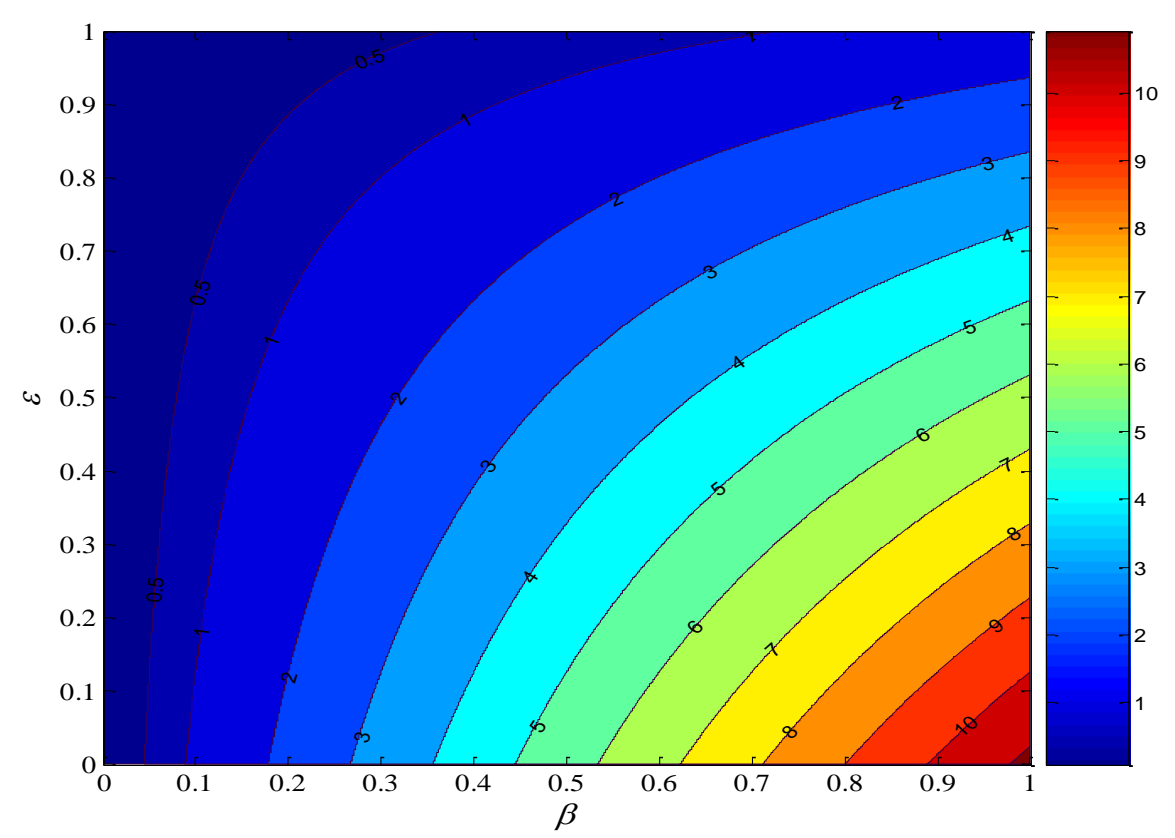

Figure 3: The impact of the disease transmission rate $\beta$ and vaccine efficacy $\varepsilon$ on $R_{V}$.

\section{Results and Discussion}

Numerical simulations using the model parameter values in Table 1 are carried out to support the theoretical results. In a circumstance where parameter values were not available in the literature, realistic values are assumed for illustration purpose. Employing the fourth and fifth order RungeKutta methods which are implemented via the ode45 function in MATLAB, the solution profiles of the model system (1) are shown in Figure 4.
The following initial conditions are used: $S(0)=10,000, V(0)=10, E(0)=10$, $A(0)=5, I_{D}(0)=5$, $I_{U}(0)=5$ and $R(0)=0$. Figure 4 shows the change in the population profiles as time increases from 0 to 300 days. During the first 70 days, the number of susceptible individuals decreases rapidly due to vaccination at a constant rate of 0.02 and through infection due to contact with infected individuals. 


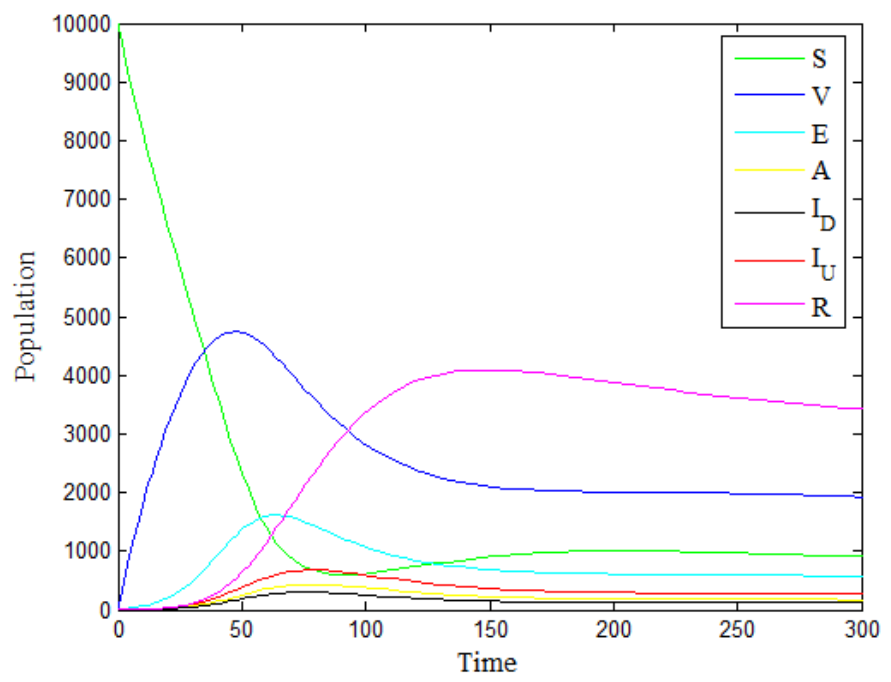

Figure 4: Evolution of population against time.

Thus, with very low new infections, the number of exposed, asymptomatic, detected, and undetected infected individuals subsequently are reduced from the $150^{\text {th }}$ day onwards and remain constant. From Figure 5, one can observe from the red curve that if vaccine and social distancing are not implemented, the basic reproduction number is greater than unity, which indicates a high possibility of COVID-19 to become endemic in the population. Social distancing was simply modelled by reducing the transmission rate, and without vaccination, the transmission rate $\beta$ must be reduced by greater than $78 \%$ to maintain the basic reproduction number less than unity. With the implementation of the vaccine at a constant rate of 0.002 and 0.005 , transmission rate must be reduced by $69 \%$ and $56 \%$, respectively to maintain the effective reproduction number less than unity.

Moreover, with the implementation of the vaccine at a constant rate of 0.02 , the transmission rate must only be reduced by $11 \%$. This implies that it is not imperative to over-implement social distancing allowing economic and social activities to function more habitually. If the vaccination rate is 0.05 (with vaccine efficacy $\varepsilon=0.8$ ), one can observe that social distancing may no longer be needed since the effective reproduction number decreases to less than unity. Thus, vaccination as an intervention has an excellent potential to allow the government to relax the social distancing intervention and to eradicate COVID-19 from the population (Aldila et al. 2021). Figure 5 depicts the effects of vaccine on sensitivity of infection rate to the effective reproduction number. 


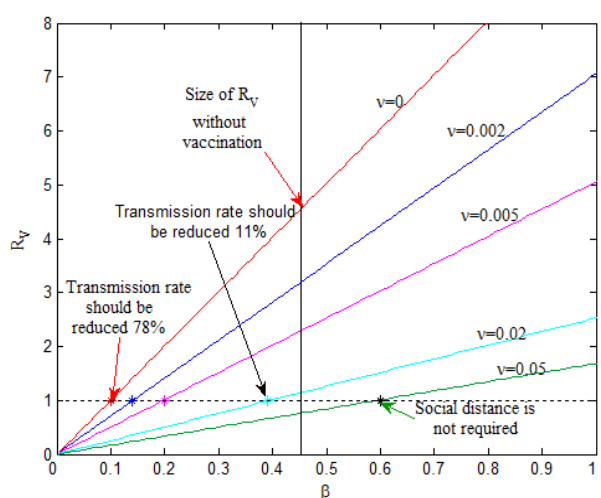

(a)

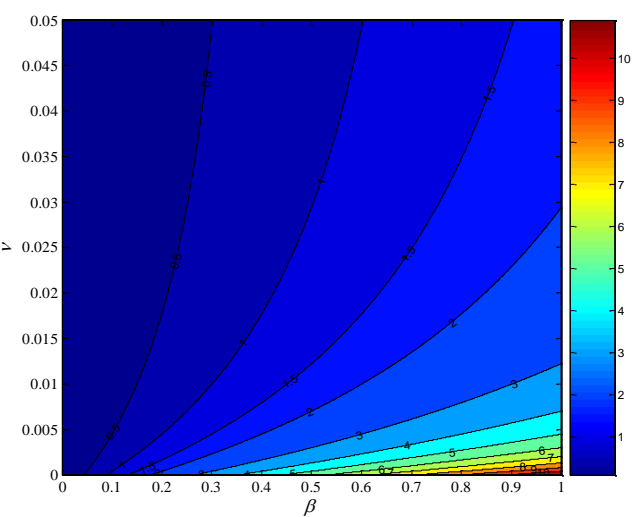

(b)

Figure 5: (a) Effect of vaccine on sensitivity of infection rate to the reproduction number $R_{V}$ (b) Contour plot of the effective reproduction number $R_{V}$ with respect to disease transmission rate $\beta$ versus vaccination rate $\nu$.

\section{Impact of detected and undetected on the COVID-19 transmission dynamics}

The impacts of detected and undetected cases are investigated when vaccination is implemented. The impact of detected and undetected cases on the effective reproduction number $R_{V}$ are presented under the range [0-1] of the proportion, $\alpha_{D}$, and $\alpha_{U}$, of the exposed individuals who progress to infectious detected and infectious undetected symptomatic individuals. As shown in Figure 6 , the graphical representation of the threshold parameter $R_{V}$ as a function of $\alpha_{D}$ and $\alpha_{U}$, the blue surface indicates the threshold $R_{V}=1$, and the red surface indicates the threshold $R_{V}=2$. The value of the basic reproduction number $R_{0}$ and effective reproduction number $R_{V}$ obtained using all parameters found in Table 1 are $R_{V}=1.451$ and $R_{0}=4.338$, respectively. Moreover, when the proportion of exposed individuals who become undetected symptomatic is 0 (i.e. $\alpha_{U}=0$ ) and the proportion of exposed individuals who become detected symptomatic is 0.7 (i. e. $\alpha_{D}=0.7$ ), the value of $R_{V}=0.629$ and $R_{0}=1.881$, respectively. In the latter case, the transmission of the epidemic could be significantly reduced with increased detection of COVID-19 cases. Moreover, $R_{V}$ decreases when the proportion $\alpha_{D}$ of detected symptomatic individuals increases. If $R_{V}$ goes below 1 , the corona virus will eventually die (Melis and Littera 2021, Samui et al. 2020). However, it is important to note that if the proportion $\alpha_{U}$ of undetected symptomatic individuals increases, the threshold $R_{V}$ increases. Therefore, the proportion $\alpha_{U}$ of undetected infectious individuals could potentially be responsible for the rapid increase of the COVID-19 epidemic (Melis and Littera 2021), and the transmission of the epidemic could be significantly reduced or halted with increased detection of COVID-19 cases. Figure 6 depicts the effective reproduction number $R_{V}$ as a function of $\alpha_{D}$, and $\alpha_{U}$ (the proportion of the exposed individuals progressing to detected infectious and undetected infectious classes). 


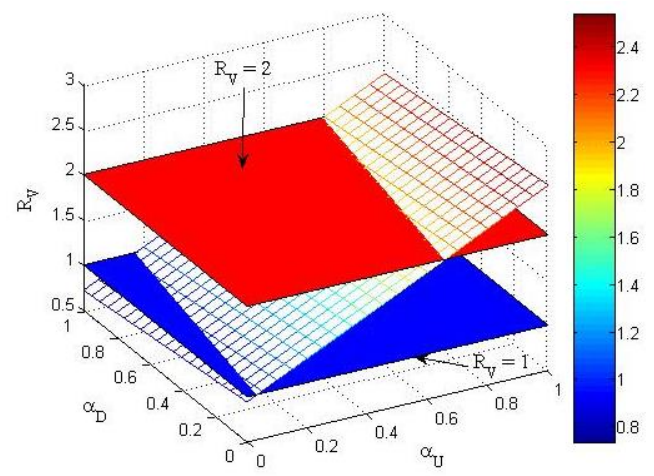

(a)

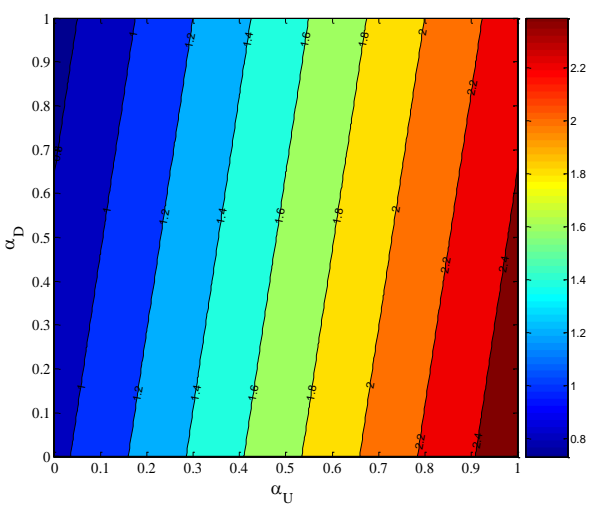

(b)

Figure 6: Effects of detected and undetected infectious symptomatic individuals to $R_{V}$.

\section{Conclusion}

A compartmental mathematical model to describe the disease transmission dynamics of COVID-19 was formulated. The model incorporates asymptomatic and symptomatic detected (identified) and undetected (unidentified) cases. The model is theoretically and numerically analyzed; its effective and basic reproduction numbers are derived. The disease-free equilibrium is both locally and globally asymptotically stable, and the disease could be eradicated when the reproduction number is below unity. The effectiveness of vaccination in minimizing the probability of disease transmission is investigated, and results show that vaccination has the potential to relax social distancing rules, while maintaining the effective reproduction number at the minimum possible and eradicate COVID-19 from the population with higher vaccination coverage.

In order to reliably detect the presence of undetected infectious individuals, it would be necessary to test the entire population and not just the symptomatic cases; however, this intervention seems not feasible to be implemented under constrained health care resources. Individuals should therefore be encouraged to report symptoms to health authorities as soon as they appear. Moreover, if the vaccine efficacy is low and the disease reproduction number is high, the disease may not be eradicated even if a large proportion of the population is vaccinated. That is, additional efforts will be needed to reduce $R_{V}$ below unity even if vaccine coverage is high. Consequently, for herd immunity, governments should encourage mass vaccination while enforcing nonpharmaceutical interventions such as face masks, hand washing and social or physical distancing measures.

\section{Acknowledgement}

The author acknowledges the anonymous reviewers for constructive comments which led to the improvement of this paper.

Declaration: The author has no conflict of interest.

\section{References}

Aldila D, Samiadji B, Simorangkir G, Khosnaw S and Shahzad M 2021 Impact of early detection and vaccination strategy in COVID-19 eradication program in Jakarta, Indonesia. BMC Res. Notes 14: 132.

Beigi A, Yousefpou A, Yasami A, GómezAguilar JF, Bekiros S, Jahanshahi H 2021 Application of reinforcement learning for effective vaccination strategies of coronavirus disease 2019 (COVID-19). Eur. Phys. J. Plus. 136: 609.

Castillo-Chavez C, Feng Z, Huang W 2002 On the computation of $R_{0}$ and its role on global stability. In: Castillo-Chavez C, 
Blower S, van den Driessche P, Kirschner D, and Yakubu AA (eds) Mathematical approaches for emerging and reemerging infectious diseases: models, methods and theory (pp. 229-250), Springer, Berlin, Heidelberg, New York.

Castillo-Chavez C and Song B 2004 Dynamical models of tuberculosis and their applications. Math. Biosci. Eng. 1(2): 361-404.

CDC 2021 COVID-19 Pandemic Planning Scenarios.

https://www.cdc.gov/coronavirus/2019ncov/hcp/planning-scenarios.html.

Accessed $23^{\text {rd }}$ September 2021.

Chinwendu EM, Dachollom S and Onwubuya IO 2020 Controlling the spread of COVID-19: Optimal control analysis. Computational and Mathematical Methods in Medicine 2020 Article ID 6862516, 14 pages.

Chitnis N, Hyman JM and Cushing JM 2008 Determining important parameters in the spread of malaria through the sensitivity analysis of a mathematical model. Bull. Math. Biol. 70: 1272-1296.

Diagne ML, Rwezaura H, Tchoumi SY and Tchuenche JM 2021 A mathematical model of COVID-19 with vaccination and treatment. Computational and Mathematical Methods in Medicine 2021 Article ID 1250129, 16 pages.

Dushoff J, Huang W, and Castillo-Chavez C 1998 Backwards bifurcations and catastrophe in simple models of fatal diseases. J. Math. Biol. 36(3): 227-248.

Hethcote HW 2000 The mathematics of infectious diseases. SIAM Rev. 42(4): 599653.

Huo Xi, Chen J and Ruan S 2021 Estimating asymptomatic, undetected and total cases for the COVID-19 outbreak in Wuhan: A mathematical modeling study. $B M C$ Infect. Dis. 21: 476.

Lakshmikantham V, Leela S and Martynyuk AA 1989 Stability Analysis of Nonlinear Systems. Marcel Dekker Inc., New York and Basel.

Mwalili S, Kimathi M, Ojiambo V, Gathungu D and Mbogo R 2020 SEIR model for COVID-19 dynamics incorporating the environment and social distancing $B M C$ Res. Notes 13: 352.

Melis M and Littera R 2021 Undetected infectives in the Covid-19 pandemic. Int. J. Infect. Dis. 104: 262-268.

Samui P, Mondal J and Khajanchi S 2020 A mathematical model for COVID-19 transmission dynamics with a case study of India. Chaos Solitons Fractals 140: 110173.

Shakhany MQ and Salimifard K 2021 Predicting the dynamical behavior of COVID-19 epidemic and the effect of controlstrategies. Chaos Solit. Fract. 146: 110823.

Tang B, Wang X, Li Q, Bragazzi NL, Tang, S, Xiao Y and Wu J 2020 Estimation of the transmission risk of the 2019-nCoV and its implication for public health interventions. J. Clin. Med. 9(2): 462.

Tchoumi SY, Diagne ML, Rwezaura H, Tchuenche JM 2021 Malaria and COVID19 co-dynamics: A mathematical model and optimal control. Appl. Math. Model. 99: 294-327.

van den Driessche P, Watmough J 2002 Reproduction numbers and sub-threshold endemic equilibria for compartmental models of disease transmission. Math. Biosci. 180(1): 29-48. 